\title{
FACTORS DETERMINING THE EFFECT OF EXERCISE ON BLOOD SUGAR IN THE DIABETIC
}

\author{
By RUSSELL RICHARDSON \\ WITH THE TECHNICAL ASSISTANCE OF \\ ALMA L. CASE \\ (From the George S. Cox Medical Research Institute, University of \\ Pennsylvania, Philadelphia)
}

(Received for publication July 27, 1934)

The work of Lawrence (1), Hetzel and Long (2) and others has established certain facts regarding the effect of exercise on blood sugar in diabetic individuals. It seemed desirable, however, to examine a larger number of such persons by means of a standard form of exercise to determine whether diabetics differ in their response to exercise and whether the severity of the diabetes influences the response. It seems certain that carbohydrate exchange is accelerated during exercise. Such acceleration should accentuate deficiencies which might pass unnoticed during the resting state.

It is commonly believed that exercise reduces the blood sugar in diabetics, though as yet too few patients have been studied to establish this as a fact. Former studies on the effects of exercise in diabetes have usually been made on a small number of patients and with a form of exercise which does not lend itself to definite measurement and standardization. Moreover, some of the forms used have been so exhausting in character as to be not suitable for the more severe diabetics. Exercise, to be most appropriate for such a study as this, must be sufficient to affect the blood sugar of normal persons and of diabetic patients in good condition and yet must not be too strenuous for those diabetic patients who may be in poor condition.

The exercise selected consisted of four five-minute periods of work on the ordinary type of rowing machine exerciser. By the use of either two or three springs a pull of 50 to 75 pounds could be provided. The rate of stroke was kept constant at forty per minute with the aid of a metronome. Between successive five-minute periods of exercise was interposed a two-minute period of rest, during which the patient remained seated on the machine. The patients were taught to flex the legs and back but not the arms. The blood for sugar determinations was taken from the arms. The external work of the summation of the pulling strokes for each half hour period was approximately $4.5 \times 10^{5}$ ergs with two springs 


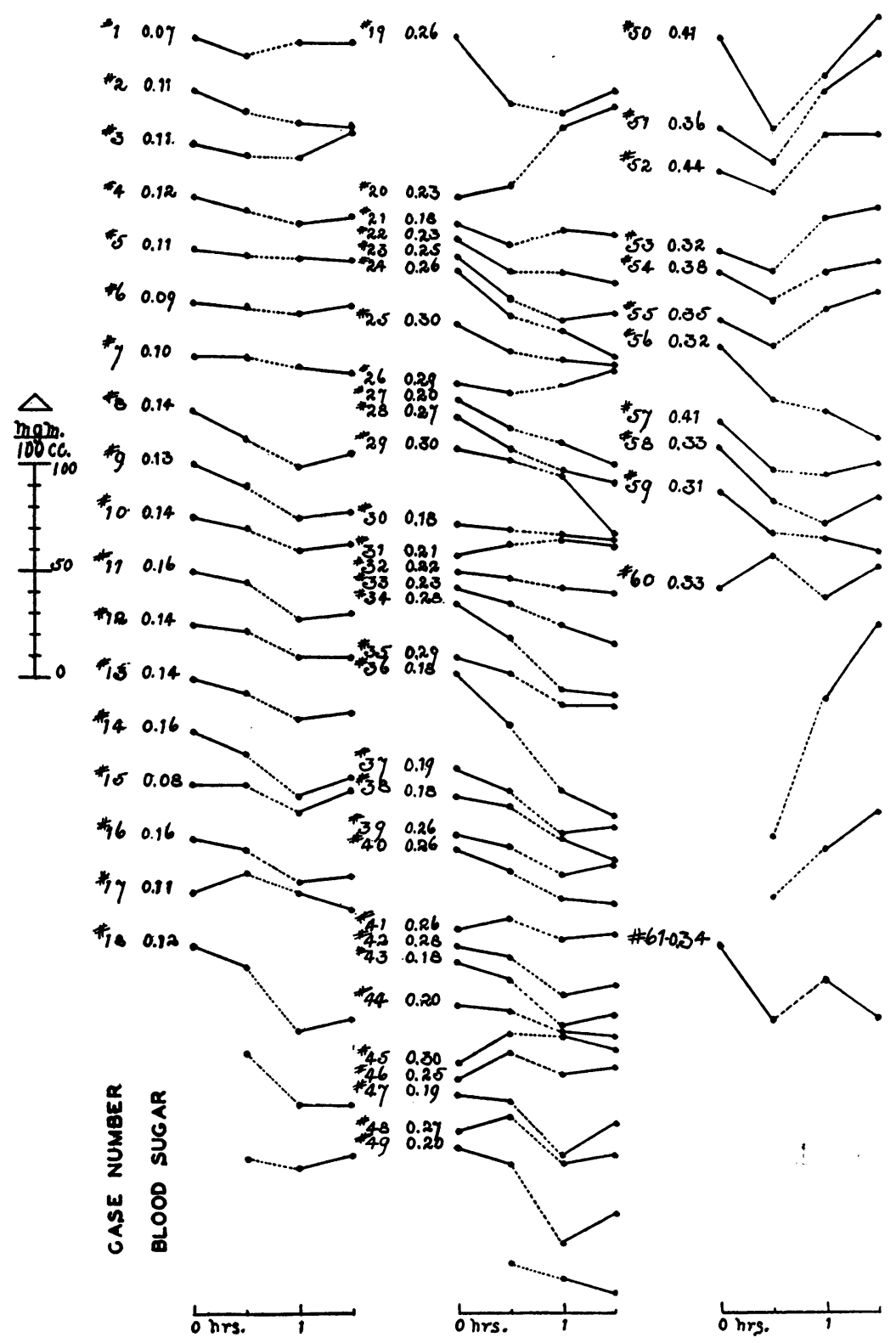

Fig. 1. Showing Blood Sugar Curves of 61 Diabetics with StaNDARDIZEd Form OF Exercise $=$ rest $\quad----.=$ exercise. 
and $6.8 \times 10^{5}$ with three springs. This exercise was found sufficient to affect the blood sugar and yet was not too exhausting for even poorly nourished patients. The subjects rested one half hour on a bed adjacent to the rowing machine immediately before and after the exercise. Neither food nor insulin had been taken for 16 hours. Blood for determination of sugar was drawn from a vein at the elbow at the beginning of the first rest period, before and after the exercise and after the second rest period. Blood sugar was measured by Benedict's method. The patients were from the metabolic clinic.

In Figure 1 are shown the blood sugar curves for 61 patients exercised in this manner. The curves are divided into three groups according to the fasting blood sugar. The first group consists of those from patients

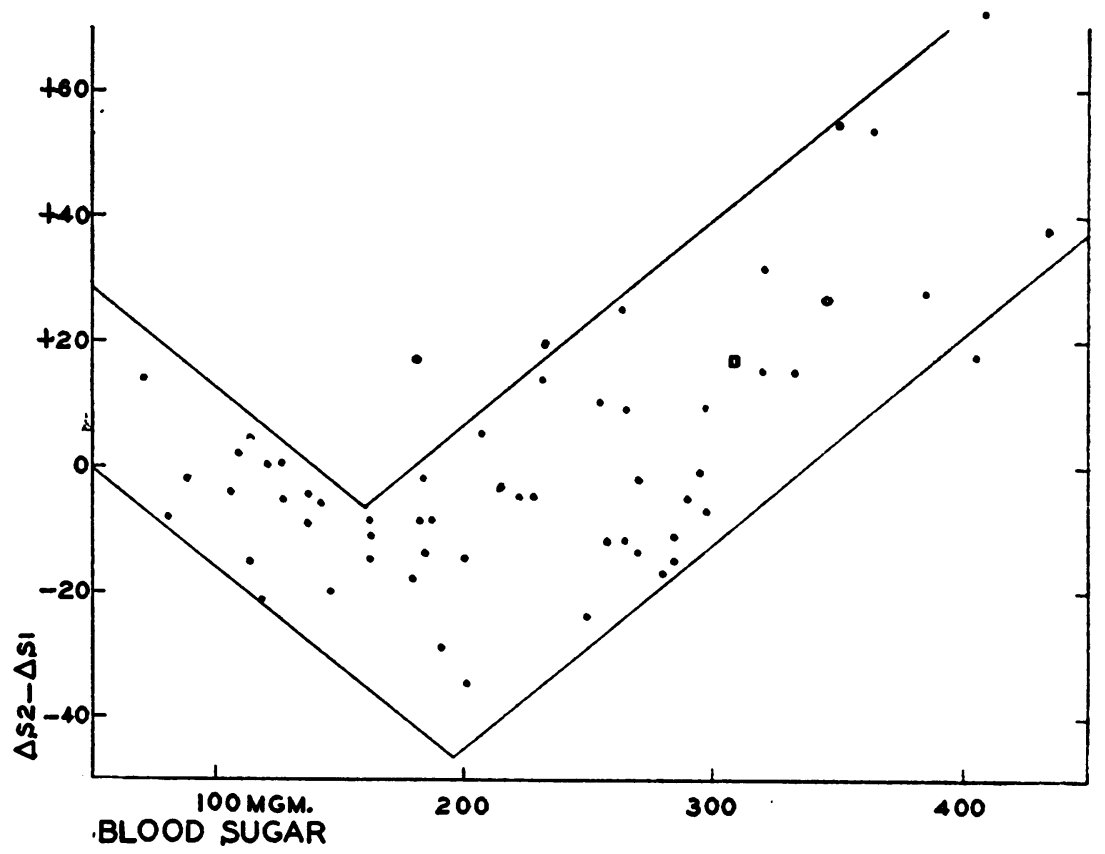

Fig. 2a. Showing the Alteration During Exercise in the Rate of Blood Sugar Change in Milligrams per Half Hour Plotted Against the Fasting Blood Sugar.

in whom the fasting blood sugar was below $175 \mathrm{mgm}$. per $100 \mathrm{ml}$. of blood, the second group from those in whom the blood sugar lay between 175 $\mathrm{mgm}$. and $300 \mathrm{mgm}$. and the third group from those in whom it was over $300 \mathrm{mgm}$. The curves are arranged in each column in the order of the change in the slope of the blood sugar curve brought about by exercise. This change in slope is expressed as $\Delta S 2-\Delta S 1$ in which $\Delta S 1$ equals the change in blood sugar during the half hour of rest preceding the exer- 
cise and $\Delta S 2$ the change in blood sugar during the half hour of exercise. A more rapid rise or less rapid fall in blood sugar results in a positive difference in slope.

In the first group the result of the exercise was predominantly a slight change in slope downward. This decrease within this group appears to be slightly greater as the fasting blood sugar was higher. In the second group, exercise induced with about equal frequency an upward or downward inflection in the course of the curve. In the third group there was an upward inflection in all patients except one. These differences are shown in Figure 2a, in which the change in the slope of the blood sugar curve is plotted against the fasting blood sugar. It will be more evident here that whereas below a fasting blood sugar of $175 \mathrm{mgm}$. the higher the blood sugar the greater the downward inflection in the curve as a result of exercise, above this fasting level the higher the fasting blood sugar the greater was the tendency to upward inflection as a result of exercise.

It appears that in the severe diabetic under the conditions of these experiments there is a tendency toward increased blood sugar as a result of exercise. This was true whether the high fasting hyperglycemia was

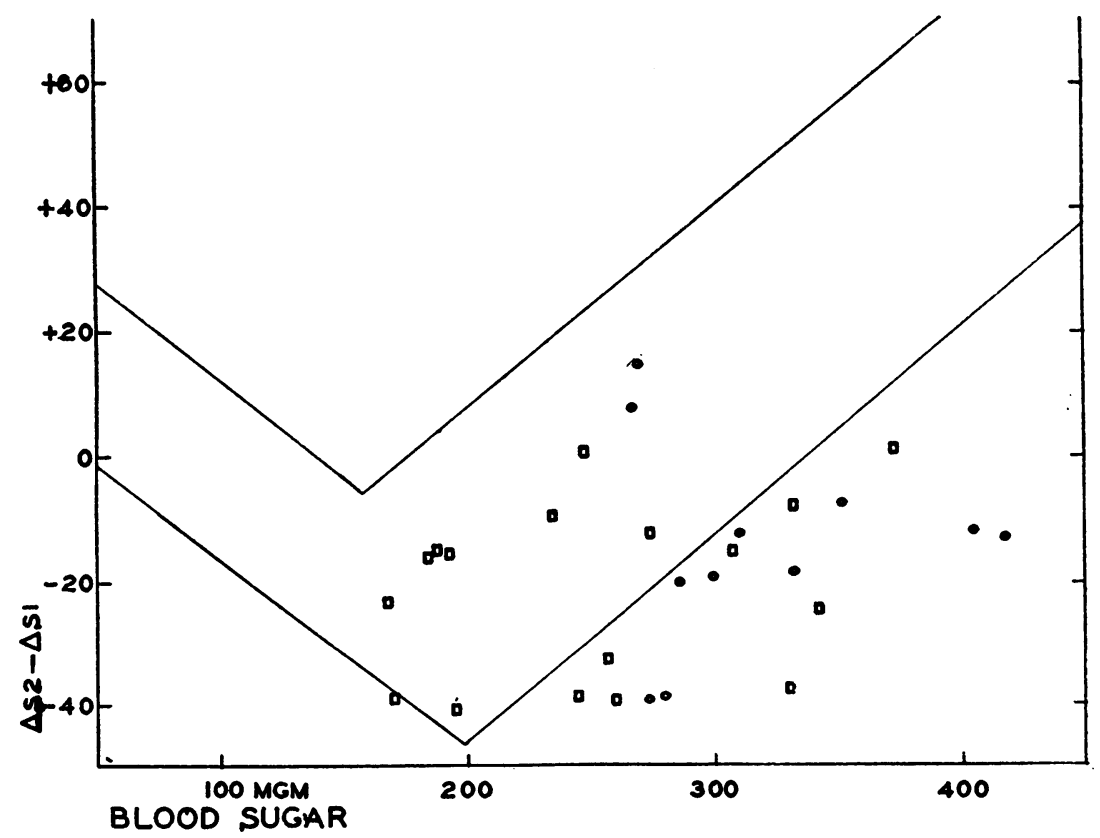

Fig. 2b. Showing the Alteration in Rate of Blood Sugar Change During Exercises Done Late in the Day as Shown in Figures 6 AND 7.

The square and circle in Figure $2 a$ show the result of exercise in the same patients without food or insulin. 
the result of a permanently severe diabetes or only a temporary condition consequent upon inadequate therapy. The patients in the former category, namely, all of those in Group 3 except the last, had been adjusted on diet and insulin and were in good condition at the time of the test. The fasting hyperglycemia had been developed as a result of 16 hours without insulin.

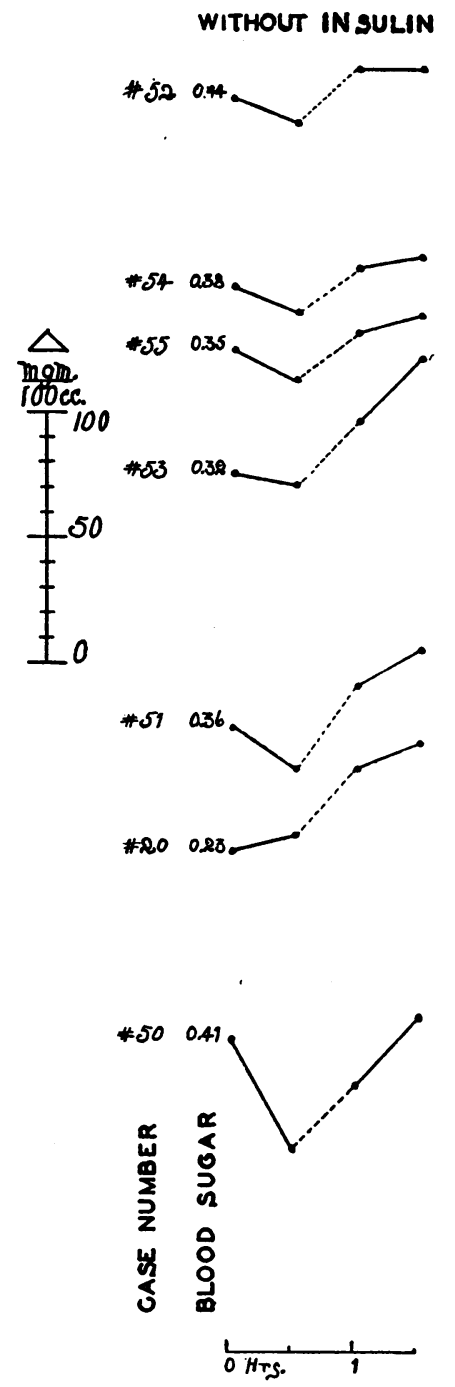

WITH INSULIN
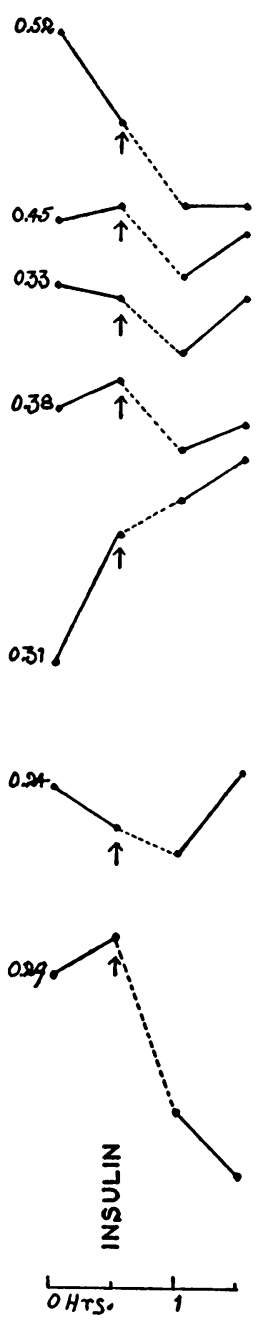

Fig. 3. Showing Effect of 0.1 Unit of Insulin Given Intravenousty IMMEDIATELY Before THE EXERCISE

The curves in the left hand column were done without insulin.

$\uparrow$ indicates 0.1 unit of insulin. 
Four patients with marked hyperglycemia on their first visit to the clinic were exercised at this visit while they were still in poor clinical condition. The blood sugar of all increased during exercise at this time. After they had been adjusted on a suitable diet with insulin, so that their fasting blood sugar was less than $120 \mathrm{mgm}$., exercise was then accompanied by a fall in blood sugar.

The use of insulin intravenously immediately before the exercise, even in very small doses, was followed by an alteration in the behavior of the blood sugar curve during exercise. In the experiments demonstrating this, variation in absorption of the insulin was eliminated by employing the intravenous route of administration; 0.1 unit was given from 2 to 3 minutes before the exercise. This amount of insulin, when not followed by exercise, had not been sufficient to cause any marked change in blood sugar. When such a dose of insulin was given to patients, who without insulin exhibited an increase in blood sugar during exercise, there resulted a definite decrease in the blood sugar during exercise. In Figure 3 are shown the blood sugar curves of seven patients exercised, first without insulin and then with insulin. It is evident that even a very small amount of insulin given intravenously immediately before exercise influenced the course of the blood sugar curve. In Case 51 it will be noted that in the experiment with insulin, though the blood sugar was slightly higher after exercise than before, still it was lower than would have been expected after rest or exercise without insulin.

It is interesting to contrast with these experiments the effect of five times the dose of insulin, given intravenously but given 30 minutes before commencing the exercise, as shown by the fourth curves from Cases 59 and 55 in Figure 4. In these experiments 0.5 unit of insulin was given intravenously after $1 / 2$ hour rest; the exercise, begun 30 minutes later, was in these experiments accompanied by a rising blood sugar. Further studies are in progress as to the significance of the difference between these two groups of experiments. When 5 units of insulin were given subcutaneously and the patient fed fillers, and exercise was begun 60 minutes later, a fall in blood sugar occurred (see first curves of Figures 6 and 7 ). It is evident that not only the size of the dose but the mode of administration, whether intravenous or subcutaneous, and the time of its administration in relation to the exercise must be taken into account.

In order to investigate the relative effectiveness of insulin combined with rest or with exercise, 8 patients, whose blood sugar had increased or had remained unchanged following exercise, were given 0.5 unit of insulin intravenously followed by 60 minutes of rest and were then given a second 0.5 unit of insulin intravenously followed immediately by the exercise. In five of these patients the decrease in blood sugar was greater after the second dose of insulin with the exercise whereas in three it was greater 
CASE NUMBER 50

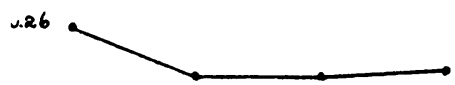

0.81
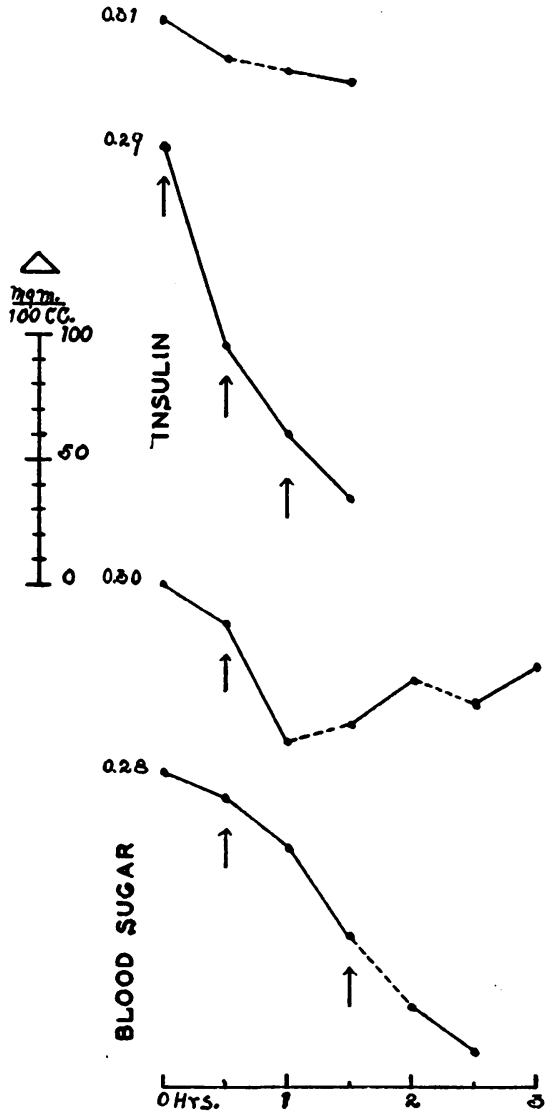

CASE NUMBER 53

0.86
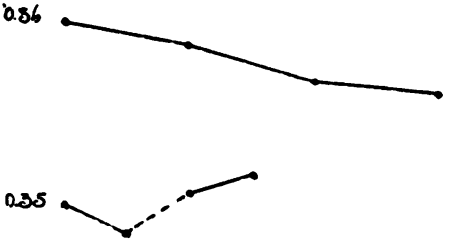

0.35
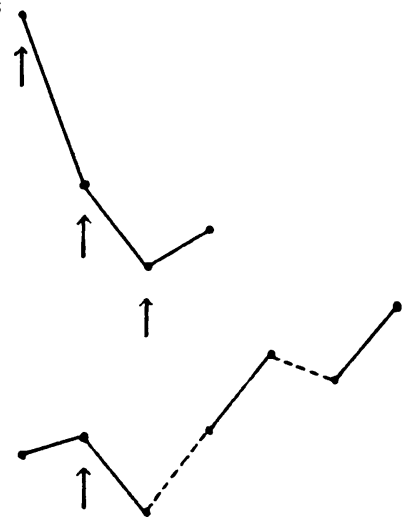

088

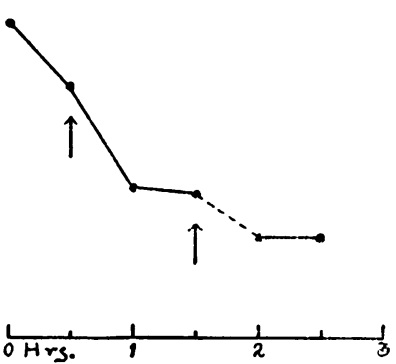

Fig. 4. Showing Effect on Blood Sugar of Various Combinations of Rest, Exercise and Insulin

$=$ rest $\quad-\quad-\rightarrow=$ exercise.

$\uparrow$ indicates 0.5 unit of insulin given intravenously.

after the first dose of insulin which was followed by rest (see Figure 5). In the former group the average decrease in blood sugar was $13 \mathrm{mgm}$. during $1 / 2$ hour of rest and $33 \mathrm{mgm}$. during $1 / 2$ hour of exercise. In the latter group the average resting decrease in blood sugar was $44 \mathrm{mgm}$. and the decrease during exercise was $25 \mathrm{mgm}$. There was no consistent effect of either rest or exercise on the blood sugar curve after this amount of insulin in these severe diabetics. 
Further observations on the blood sugar accompanying various combinations of insulin, rest and exercise are shown by the curves in Figure 4. These were done on Cases 55 and 59 who have diabetes of such a degree that 80 and 65 units of insulin respectively are required daily with a maintenance diet.

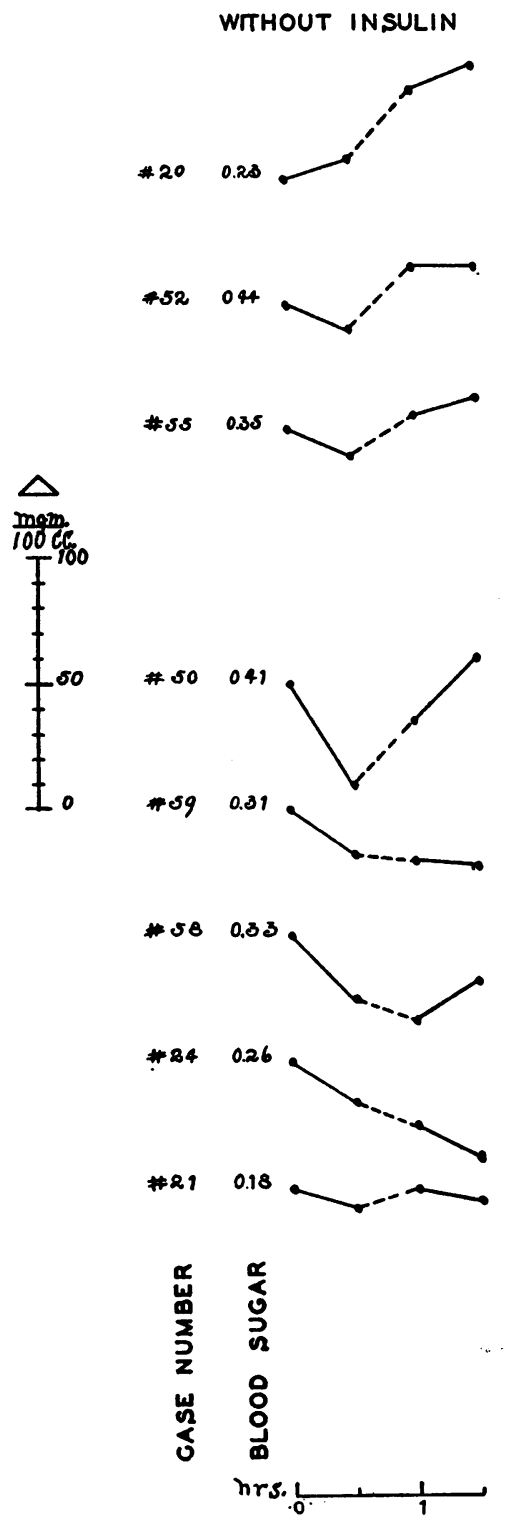

WITH INSULIN

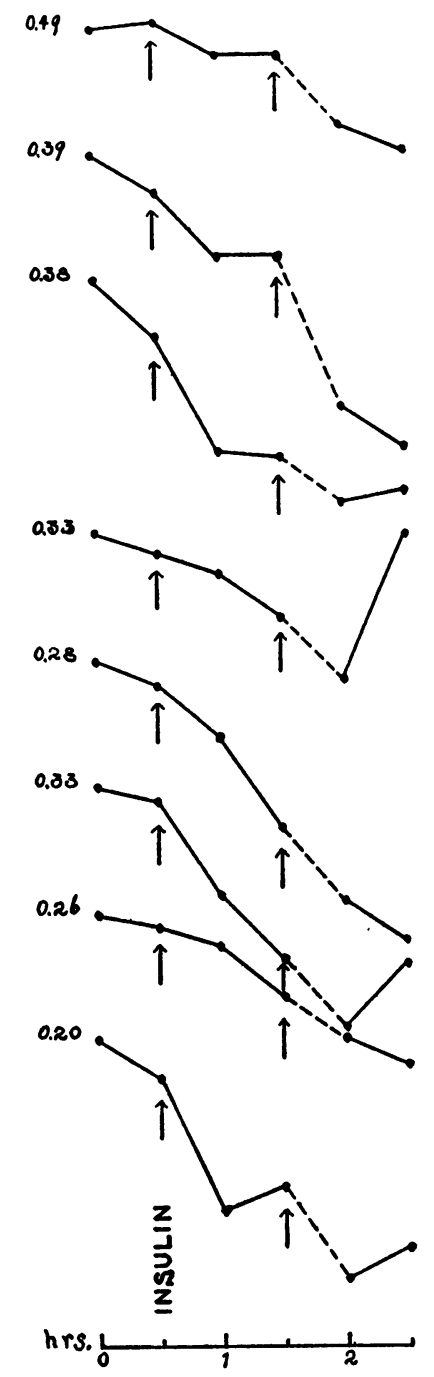

Fig. 5. Showing Effect on Blood Sugar of Insulin Followed by Rest AND BY EXERCISE

indicates 0.5 unit insulin intravenously. 

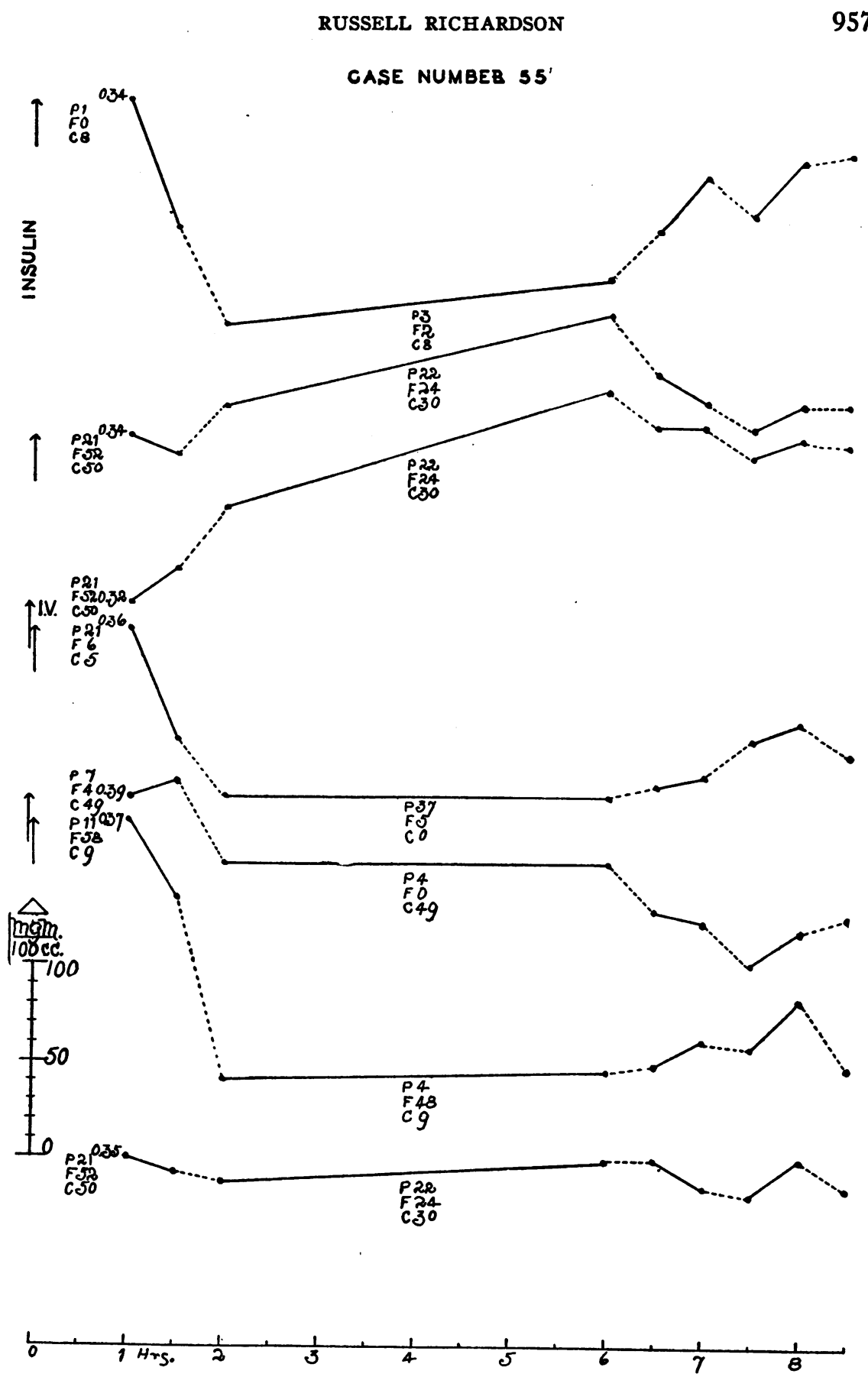

Fig. 6. Showing Blood Sugar Curves of All Day Experiments on Patient 55

$\rightarrow=$ rest $\quad---\rightarrow=$ exercise.

$\uparrow$ indicates 5 units insulin. 
The first curves from each patient confirm the observation previously made, that during rest without food or insulin there is little change in blood sugar.

It will be noted that there is a considerable similarity in blood sugar changes at corresponding periods on different days; thus the resting periods in curves number $1,2,4$, and 5 are quite consistent, as also the responses to insulin during the second periods in curves number 3,4 , and 5 .

In order to study the effect of food in association with insulin and exercise a longer experiment was performed which included insulin, two meals and four periods of exercise, the whole extending over $8 \frac{1}{2}$ hours. These experiments also were carried out on Cases 59 and 55. The food was varied, including a day with a low intake, a day with an average diabetic diet and one day each on which protein, carbohydrate and fat respectively comprised the bulk of the diet. Five units of insulin were given subcutaneously on each day except two. On one of these no insulin was given and on the other 5 units were given intravenously. In Figures 6 and 7 are shown the blood sugar curves for these days.

Several points are worthy of note. The similarity in response to intravenous and subcutaneous insulin is evident. Protein and fat seemed to provide no immediately available effect to offset the action of the insulin. The exercises later in the day are of interest. It had been shown that these patients when fasting had an increased blood sugar after exercise. The decrease in blood sugar with all periods of exercise later in the day in these experiments suggests either that some of the small dose of insulin given at the beginning of the day still remained available or that food intake acted in some way to reduce blood sugar during exercise. On the last day no insulin was given and yet the same reduction in blood sugar occurred. This fact seems to show that the insulin given in the morning was not the deciding factor. It appears that intake of food provided a condition which, with exercise, resulted in reduction of blood sugar (see Figures 6 and 7, curves 7, and Figure 2b).

\section{DISCUSSION}

The antagonistic action of insulin and adrenalin in controlling blood sugar is established. There are, in addition, two other factors which may at times affect this control so that, in all, four factors may be said to govern the blood sugar in exercise. These are: (1) Adrenalin raising the blood sugar. (2) Insulin lowering the blood sugar. (3) The amount of glycogen in the muscles as affected by the exercise itself- " muscle glycogen vacuum" so called. (4) The amount of glycogen in the liver available for supplying sugar to the blood.

In the normal individual exercise reduces the amount of glycogen in the muscles and there is created a demand for glucose at this site. This 

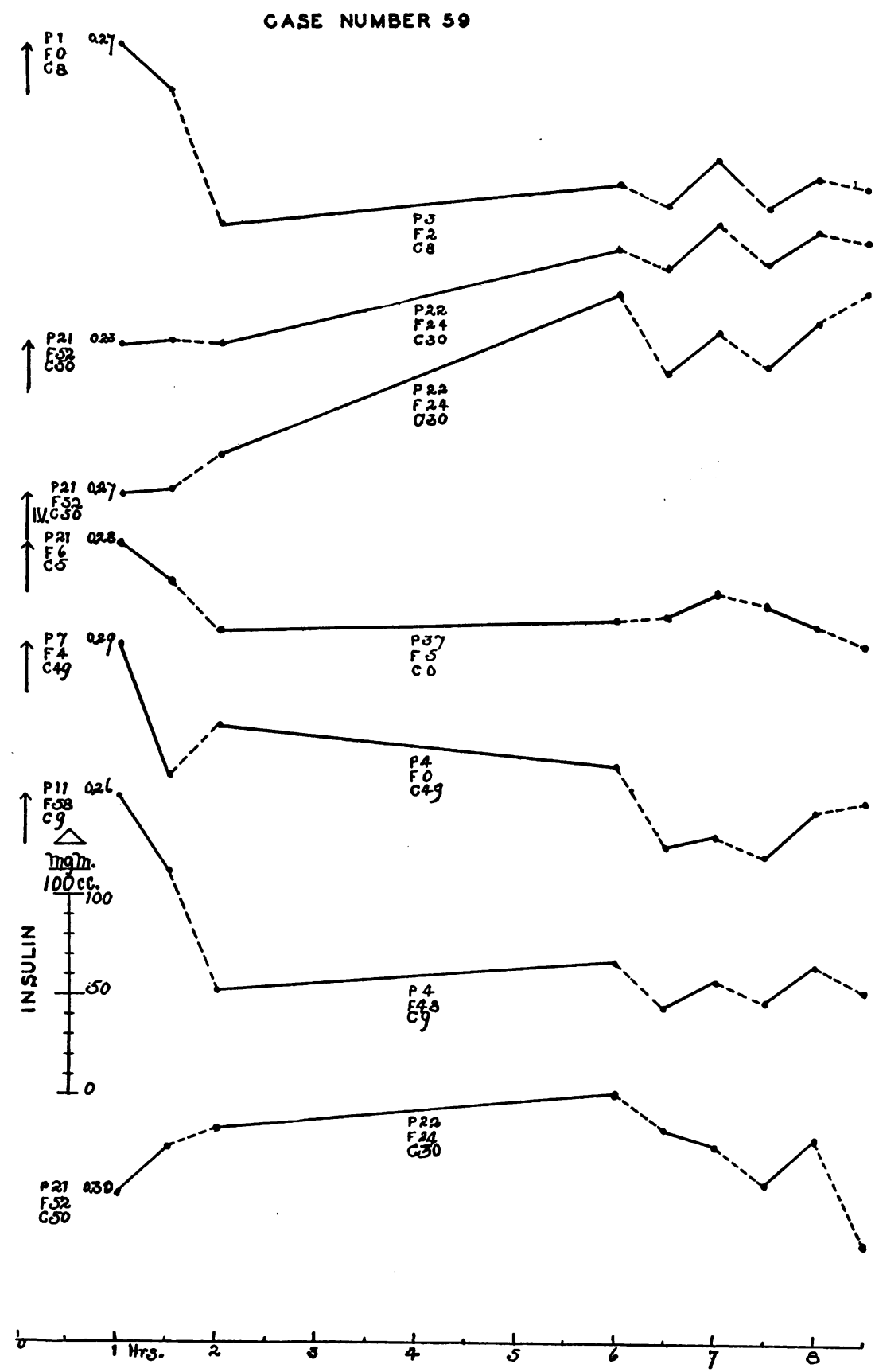

Fig. 7. Showing Blood Sugar Curves of All Day Experiments on Patient 59

$=$ rest $\quad---\rightarrow=$ exercise.

$\uparrow$ indicates 5 units insulin. 
has been called the "muscle glycogen vacuum." Exercise probably excites an increased availability of both adrenalin and insulin, thereby supplying more glucose to the blood and converting it in turn to the use of the exercising muscles.

Excessive exercise in the normal subject may be accompanied by a marked hypoglycemia. This is usually attributed to a depletion of the stores of glycogen in the body. In the diabetic the glycogen of both muscles and liver is usually lower than normal. With this low glycogen content of the tissues exhaustion of the stores might be expected to occur more readily and this is a plausible explanation of the tendency to hypoglycemia after exercise in the mild diabetics who are still able to withdraw glucose from the blood during exercise.

When the diabetes is so mild that a normal blood sugar follows a 16hour fast, the blood sugar curve after exercise is apparently normal. There is only a slight change in blood sugar with exercise and that change is usually downward. It appears that the normal protection against marked hypoglycemia is still effective in these patients.

With a little less mild diabetes, indicated by a slightly higher fasting blood sugar, a greater drop occurs after exercise. The blood sugar seems to show the greatest decrease when it starts from approximately $175 \mathrm{mgm}$. per $100 \mathrm{ml}$. of blood. It may be presumed that in such cases the stimulus of exercise makes demands upon the blood sugar which the mechanisms or the reserves of the body can not quite adequately support.

As the ability of the body to use carbohydrate becomes still further impaired a different factor predominates. The stimulus of exercise seems to lead to less withdrawal of blood sugar. Presumably this is due to deficient insulin. Little or no change occurs in the blood sugar level during exercise. This behavior was usually observed with a fasting blood sugar between 200 and $300 \mathrm{mgm}$.

When the diabetes is of sufficient degree so that the fasting blood sugar is above $300 \mathrm{mgm}$. the reduction in the ability to withdraw sugar from the blood was, in almost all the severe diabetics studied, so marked that in these individuals the blood sugar actually rose during a $1 / 2$ hour period of exercise as is shown in Figure 1, third column. After only 5 minutes of exercise there was a definite decrease in the blood sugar in some of the severe diabetics, but after a further period of exercise the increase occurred. It would appear, therefore, that in some patients there was a sharply limited capacity for withdrawing blood sugar which was exhausted during the first few minutes of exercise. If the decreases in blood sugar following exercise are dependent on available insulin then the food intake in the day in Cases 59 and 55 appears to have increased the availability of the patients' insulin; for no insulin had been taken by these patients for almost 24 hours (see Figures 6 and 7, last curves). 
Comparisons between the effects of exercise on blood sugar in the mild and in the severe diabetic suggest that, in the former, the difficulty is one of inadequate or inadequately available glycogen reserves and, in the latter, a more or less complete inability to convert glucose in the blood to the needs of the tissues.

\section{CONCLUSIONS}

A standard form of exercise has been used with 61 diabetic patients with blood sugar determinations made before and after the period of exercise.

The effect of exercise of the degree and character employed in these experiments, after 16 hours without food or insulin, varied with the severity of the diabetes as indicated by the fasting blood sugar level.

With increase in the fasting blood sugar level from a normal value to about $175 \mathrm{mgm}$. per $100 \mathrm{ml}$. of blood the effect of a half hour of standardized exercise induced a progressively more marked lowering of the blood sugar.

With further increase in the fasting blood sugar level from $175 \mathrm{mgm}$. to above $300 \mathrm{mgm}$. the effect of a half hour of standardized exercise induced a progressively more marked elevation of the blood sugar.

In the severe diabetic the intravenous injection of 0.1 unit of insulin, which given before a period of rest was without marked effect on the blood sugar, caused, when given immediately before a period of exercise, a recognizable drop in blood sugar. Five times this dose given intravenously a half hour before beginning the exercise failed to prevent in the severe diabetic this rise associated with the exercise.

The effect of 0.5 to 5 units of insulin subcutaneously was not influenced by rest or exercise.

Recent intake of food led under certain conditions to a decrease in blood sugar during exercise in two diabetics, who, fasting, exhibited a rise of blood sugar during exercise.

The author wishes to acknowledge with thanks suggestions by Dr. J. Harold Austin.

\section{BIBLIOGRAPHY}

1. Lawrence, R. D., The effect of exercise on insulin action in diabetes. Brit. M. J., 1926, 1, 648.

2. Hetzel, K. S., and Long, C. N. H., The metabolism of the diabetic individual during and after muscular exercise. Proc. Roy. Soc., B, 1926, 99, 279. 